\title{
Inestabilidades dinámicas en cerámicas monocristalinas a base de circona
}

\author{
A. GALLARDO-LÓPEZ, D. GÓMEZ-GARCíA Y A. DOMÍNGUEZ-RODRÍGUEZ, L. KUBIN ${ }^{+}$ \\ Departamento de Física de la Materia Condensada-Universidad de Sevilla-4180, Sevilla, España \\ ${ }^{+}$Laboratoire d'Etude des Microstructures, LEM-CNRS/ONERA, 29 Av. de la Division Leclerc, BP 72, 92322 Châtillon Cedex, France
}

\begin{abstract}
Las cerámicas monocristalinas han sido objeto de un considerable interés a lo largo de estos años, debido a la posibilidad de obtener información esencial sobre propiedades fundamentales que puedan ayudar a la comprensión de sistemas policristalinos complejos. La circona dopada con itria ha merecido un estudio particular, debido a los interesantes fenómenos que manifiesta (endurecimiento por presencia de soluto disuelto en la red, precipitación de segundas fases, etc.). Entre las propiedades menos estudiadas hasta ahora se encuentran las inestabilidades plásticas que se presentan en la deformación a alta temperatura de monocristales con alto dopado de itria. Este trabajo recoge algunas de las experiencias mecánicas que se han realizado de forma sistemática para la comprensión del fenómeno y de las condiciones requeridas para que éste se produzca. Se han realizado ensayos de deformación en compresión uniaxial a lo largo de la dirección cristalográfica $<112>$, de monocristales de circona completamente estabilizada con itria $\left(\geq 21 \mathrm{~mol} \%\right.$ ), a temperaturas entre 1310 y $1410{ }^{\circ} \mathrm{C}$. La modelización de estas inestabilidades ha requerido un análisis detallado mediante microscopía electrónica de transmisión de la estructura de dislocaciones activadas durante la deformación.
\end{abstract}

Palabras clave: YCSZ, monocristal, propiedades mecánicas, inestabilidades plásticas, efecto Portevin-le Chatelier.

\section{Dynamic instabilities in zirconia-based single- crystalline ceramics}

Single-crystalline ceramics have been the object of a considerable interest over the past years because they offer the possibility of obtaining essential information on fundamental properties which may help us to understand complex polycrystalline systems. Yttria-doped zirconia has deserved a particular study, since it exhibits quite interesting properties (solute hardening, precipitation hardening, etc.). Amongst the less studied properties up to now, plastic instabilities are found. These instabilities occur at high temperature deformation in highly-doped yttria-zirconia single crystals. This work summarizes some of the mechanical tests carried out systematically to understand this phenomenon and the conditions required for its occurrence. Yttria fully-stabilized cubic zirconia single crystals ( $\geq 21 \mathrm{~mol} \%$ yttria content) have been deformed plastically under uniaxial compression in an $<112>$ axis, in the temperature range $1310-1410^{\circ} \mathrm{C}$. Modelling of these instabilities has also required a thorough analysis by transmission electron microscopy of the deformed samples.

Keywords: YCSZ, single crystal, mechanical properties, plastic instabilities, Portevin-le-Chatelier effect.

\section{INTRODUCCIÓN}

De entre las cerámicas avanzadas, la solución sólida de circona-itria $\left(\mathrm{Y}_{2} \mathrm{O}_{3}-\mathrm{ZrO}_{2}\right)$ ha recibido una gran atención en el ámbito científico debido a sus excelentes cualidades, en particular a sus propiedades mecánicas a altas temperaturas. Dependiendo del contenido en itria, pueden desarrollarse distintas microestructuras con propiedades mecánicas muy diversas. Para bajas concentraciones de itria y en estado policristalino, tiene un comportamiento superplástico (1), propiedad muy importante en el conformado y en la unión cerámica $(2,3)$. Motivado por estas propiedades, se ha iniciado en nuestro grupo el estudio de policristales con tamaño de grano en el rango nanométrico; nuevas propiedades han aparecido, lo que abre un campo muy importante en el área de las nanocerámicas (4). Para estas concentraciones y en su estado monocristalino, dependiendo de tratamientos térmicos, presenta un endurecimiento y ablandamiento por interacción dislocación-precipitado tetragonal $(5,6)$, y ya en su fase cúbica monocristalina (concentración de itria $>9 \mathrm{~mol} \%$ ) (YCSZ) presenta un comportamiento de deformación-restauración, típico de deformación a altas temperaturas $(7,8)$.
En estos monocristales cúbicos con alto contenido en itria, se ha encontrado (9) un comportamiento anómalo en el régimen de deformación plástica, con oscilaciones de "dientes de sierra" en las curvas fuerza (tensión)-deformación, bajo ciertas condiciones de temperatura y velocidad de deformación. Este fenómeno fue explicado en términos de un "ablandamiento" térmico localizado en la banda de deslizamiento, usando un modelo de inestabilidades plásticas tipo T (10). En este trabajo pondremos de manifiesto que un modelo de este tipo no resulta satisfactorio para explicar las anomalías observadas. Mediante el estudio de estas inestabilidades dinámicas y de las condiciones que las provocan, llegaremos a una explicación física plausible para este hecho experimental.

\section{PARTE EXPERIMENTAL}

Monocristales de YCSZ con 21, 24 y $28 \mathrm{~mol} \%$ de itria fueron orientados mediante la técnica de Laue, de rayos $X$ retrodispersados. Una vez orientados, se cortaron paralelepípedos 
de $2 \times 2 \times 5 \mathrm{~mm}$, de tal forma que el eje mayor coincidiera con la dirección cristalográfica [1 $\overline{1}$ ] $]$, y sus caras laterales con los planos cristalográficos (110) y (1 11$)$ respectivamente. La orientación escogida favorece el deslizamiento simple, en el plano de deslizamiento primario (001) con lo que se evita la interacción de dislocaciones de distintos planos de deslizamientos; al menos en los primeros estadios de la deformación plástica; por lo que la consolidación y las inestabilidades se deben esencialmente a la interacción dislocación- defectos puntuales.

Las muestras fueron cortadas con discos adiamantados y en algunas muestras se pulieron las caras laterales con pasta de diamante hasta $1 \mu \mathrm{m}$ antes de ser sometidas a ensayos mecánicos de compresión uniaxial a alta temperatura (1310$1410{ }^{\circ} \mathrm{C}$ ) con velocidad de traversa constante. Se usaron equipos comerciales Instron (mod. 1185 y 1586). Las velocidades de desplazamiento de traversa impuestas equivalen a velocidades de deformación entre $3 \cdot 10^{-4}$ y $6 \cdot 10^{-3} \mathrm{~m} \cdot \mathrm{s}^{-1}$. Para intentar establecer los límites de las inestabilidades dinámicas, se hicieron también recocidos, cargas y descargas de las muestras, y cambios de temperatura y velocidad de deformación.

La superficie de las muestras deformadas fue observada mediante microscopía óptica para determinar la existencia de bandas de deslizamiento. Finalmente, la microestructura de las muestras deformadas fue investigada mediante microscopía electrónica de transmisión (MET), para lo cual se cortaron y adelgazaron láminas orientadas según el plano primario de deslizamiento (001). Las muestras fueron observadas en un microscopio electrónico Hitachi H-800 a 200 kV (Servicio de Microscopía Electrónica de la Universidad de Sevilla).

\section{RESULTADOS}

\subsection{Condiciones para las inestabilidades dinámicas.}

En la tabla I se exponen las condiciones de velocidad y temperatura de los experimentos de deformación plástica en los que se produjeron inestabilidades, comparándolos con datos de la literatura (11). Se muestran los datos más significativos de entre los 20 ensayos de deformación realizados.

De la tabla I pueden deducirse varias características de las inestabilidades dinámicas. En el intervalo de velocidades de deformación experimental, existe un límite superior de temperatura. En las muestras de 21, 24 y $28 \mathrm{~mol} \%$ deformadas a $1,6 \cdot 10^{-5} \mathrm{~s}^{-1}$ no hemos observado inestabilidades por encima de los $1380^{\circ} \mathrm{C}$. Existen pares de valores temperatura-velocidad de deformación muy concretos para los cuales se producen las inestabilidades. Debemos también tener en cuenta que un aumento de la velocidad de deformación plástica tiene un efecto similar al de una disminución de la temperatura.

\subsection{Características de las inestabilidades dinámicas.}

Se dan al inicio de la deformación plástica. Desaparecen tras un intervalo de deformación, típicamente del $8 \%$. Su amplitud es variable, entre 1 y $20 \mathrm{MPa}$, dependiendo de las condiciones $(\dot{\varepsilon}, \mathrm{T})$, al igual que su frecuencia que oscila entre 0,01 y 0,08 Hz. Consisten en una caída brusca de tensión (que se produce durante un intervalo $t \sim 1$ s) seguida de un aumento progresivo de dicha tensión (recuperación aparentemente elástica), tal como puede verse en la figura 1. Esta figura muestra un experimento con varias etapas (cambios de temperatu-
TABLA I.- CONDICIONES EXPERIMENTALES DE TEMPERATURA Y VELOCIDAD DE DEFORMACIÓN EN QUE SE HAN PRODUCIDO INESTABILIDADES DINÁMICAS. ESTUDIO COMPARATIVO ENTRE DATOS DE (11) Y DE ESTE ESTUDIO. LOS DATOS EN ITÁLICA CORRESPONDEN A (11), MIENTRAS QUE EL RESTO SON ORIGINALES DE ESTE TRABAJO.

\begin{tabular}{|c|c|c|c|}
\hline $\begin{array}{c}\mathrm{Y}_{2} \mathrm{O}_{3} \\
(\mathrm{Mol} \%)\end{array}$ & Temperatura $\left({ }^{\circ} \mathrm{C}\right)$ & $\begin{array}{c}\text { Velocidad de } \\
\text { deformación, } \dot{\varepsilon}\left(\mathrm{s}^{-1}\right)\end{array}$ & $\begin{array}{c}\text { Tensión inicio } \\
\text { serraciones (MPa) }\end{array}$ \\
\hline 18 & $\begin{array}{l}1200 \\
1300\end{array}$ & $\begin{array}{l}1,2 \cdot 10^{-5} \\
2,4 \cdot 10^{-5}\end{array}$ & $\begin{array}{l}370 \\
340 \\
330\end{array}$ \\
\hline 21 & $\begin{array}{l}1400 \\
1500 \\
1380\end{array}$ & $\begin{array}{l}1,2 \cdot 10^{-5} \\
1,2 \cdot 10^{-4}\end{array}$ & $\begin{array}{l}350 \\
330 \\
330 \\
370\end{array}$ \\
\hline 24 & $\begin{array}{l}1310 \\
1320 \\
1330 \\
1340 \\
1350\end{array}$ & $\begin{array}{l}1,6 \cdot 10^{-5} \\
\\
3,2 \cdot 10^{-5} \\
6,4 \cdot 10^{-5} \\
1,6 \cdot 10^{-4}\end{array}$ & $\begin{array}{l}390 \\
375 \\
370 \\
365 \\
365 \\
348 \\
352 \\
371 \\
415\end{array}$ \\
\hline 28 & $\begin{array}{l}1330 \\
1345 \\
1350\end{array}$ & $1,6 \cdot 10^{-5}$ & $\begin{array}{l}350 \\
340 \\
330\end{array}$ \\
\hline 32 & 1200 & $1,2 \cdot 10^{-5}$ & 370 \\
\hline
\end{tabular}

TABLA II.- CARACTERÍSTICAS DE LAS INESTABILIDADES DINÁMICAS DEL ENSAYO DE LA FIGURA 1.

\begin{tabular}{|c|c|c|c|}
\hline Temperatura $\left({ }^{\circ} \mathrm{C}\right)$ & $\mathrm{n}^{\mathrm{o}}$ & Amplitud media $(\mathrm{MPa})$ & Duración media (s) \\
\hline 1350 & 5 & $12 \pm 4$ & $80 \pm 30$ \\
\hline 1340 & 18 & $8 \pm 4$ & $36 \pm 11$ \\
\hline 1330 & 10 & $10 \pm 6$ & $43 \pm 13$ \\
\hline 1320 & 15 & $10 \pm 6$ & $40 \pm 20$ \\
\hline $1310-1$ & 5 & $5 \pm 3$ & $24 \pm 12$ \\
\hline $1310-2$ & 18 & $1,8 \pm 0,8$ & $12 \pm 5$ \\
\hline
\end{tabular}

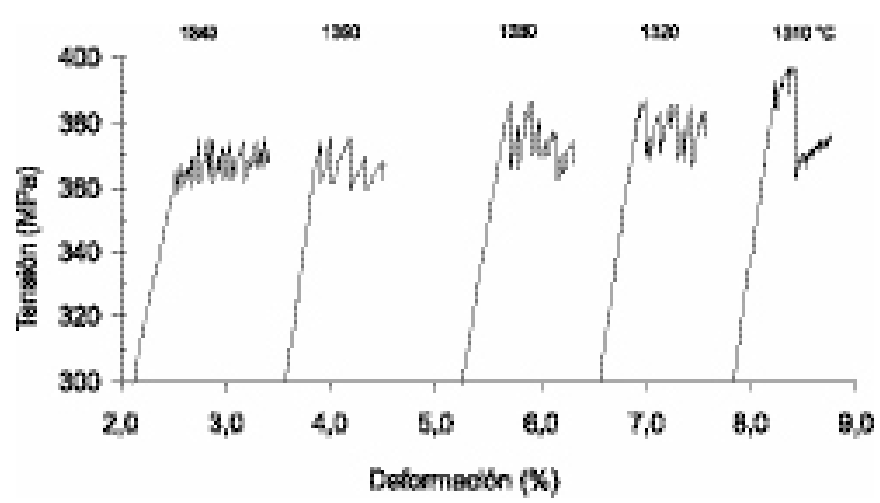

Fig. 1.- Curva tensión-deformación con inestabilidades plásticas de una muestra con $24 \mathrm{~mol} \% \mathrm{Y}_{2} \mathrm{O}_{3}$, a velocidad de traversa de $5 \cdot 10^{-4} \mathrm{~m} \mathrm{~s}^{-1}$. Se muestran distintos tramos con temperaturas entre 1310 y $1350^{\circ} \mathrm{C}$.

ra) donde son claramente visibles las inestabilidades en cada tramo. La tabla II recoge características de estas oscilaciones. La recuperación elástica de tensión (aumento de la tensión con pendiente constante) se transforma en una recuperación plástica cuando aumentamos la temperatura hasta el límite superior para las inestabilidades (ver tramo a $1350^{\circ} \mathrm{C}$ de la fig. 1). Una de las características clave de las inestabilidades observadas es que los cambios de temperatura o de velocidad 
de deformación, previa descarga de la muestra, no afectan a la tensión. Esto puede apreciarse también en la figura 1. La única consecuencia de los cambios de temperatura y velocidad de deformación es la modificación de la frecuencia y amplitud de las oscilaciones, y no el cambio de tensión que en circunstancias normales se produciría. $\mathrm{Ni}$ el aumento ni la disminución de la temperatura o la velocidad de deformación afectan a la tensión de límite elástico en el régimen inestable.

\subsection{Recocidos.}

Los recocidos sin carga de muestras durante intervalos de tiempo entre 0,5 y $2,5 \mathrm{~h}$ realizados bien sea tras tramos de deformación inestable, bien tras tramos con deformación normal (véase fig. 2), producen incrementos de la tensión de límite elástico del orden de $20 \mathrm{MPa}$. Estos picos de tensión tan acusados no se observan al inicio de la deformación plástica de las muestras en bruto. La figura 2 muestra una curva tensión-deformación para una temperatura baja, $\left(1310^{\circ} \mathrm{C}\right)$ en la que no se producen inestabilidades, y sin embargo sí se aprecian estos incrementos de tensión tras los recocidos. Tras el incremento inicial, la tensión vuelve al nivel que tenía antes del recocido en régimen estacionario.

\subsection{Estudio macroscópico de las muestras deformadas.}

Las muestras deformadas fueron observadas por microscopía óptica, mostrando en las caras laterales escalones producidos por bandas de deslizamiento en las que se producen avalanchas de dislocaciones. Estas bandas corresponden al plano de deslizamiento primario, (001). Sin embargo, a esta escala no se han encontrado diferencias marcadas entre las muestras con deformación inestable y aquéllas con una deformación normal.

\subsection{MET de las muestras deformadas. Subestructura de dis- locaciones.}

Se observa una diferencia clara entre la subestructura de dislocaciones de muestras poco deformadas (hasta el límite elástico) y otras con un mayor grado de deformación. En las muestras poco deformadas (figura 3), las dislocaciones son mayoritariamente rectas y paralelas entre sí, aunque también se detecta un número considerable de bucles en el plano primario. La densidad de dislocaciones se ha estimado en torno a $10^{13} \mathrm{~m}^{-2}$. El vector de Burgers de las dislocaciones está orientado según [1 10 ], tal y como se esperaba, ya que corresponde a la activación del sistema primario.

La micrografía de la figura 4 pertenece al mismo plano de una muestra deformada hasta el $8 \%$, que sufrió inestabilidades que después cesaron, y muestra una subestructura tridimensional de dislocaciones, o "bosque". Son dislocaciones más largas y curvadas, y su distribución indica una gran interacción entre ellas. La densidad es ligeramente superior a la muestra con menor deformación. No se observan precipitados ni otros defectos en las muestras deformadas. Tampoco existen diferencias apreciables con muestras que no sufrieron inestabilidades plásticas.

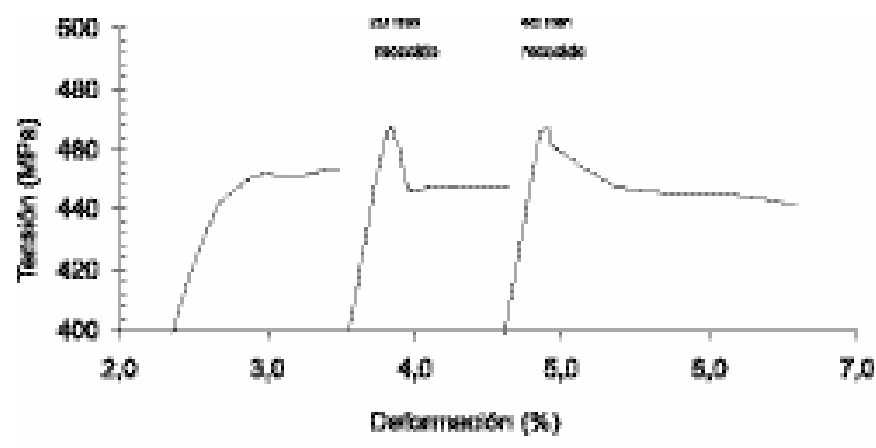

Fig. 2.- Curva tensión-deformación de una muestra con 24 mol\% $\mathrm{Y}_{2} \mathrm{O}_{3}$ fuera del régimen de inestabilidades, a velocidad de traversa $5 \cdot 10^{-4} \mathrm{~m}$ $\mathrm{s}^{-1}$, y temperatura $1310^{\circ} \mathrm{C}$, mostrando descargas seguidas de recocido durante distintos intervalos de tiempo. Se observa un incremento de tensión al comienzo de cada tramo.

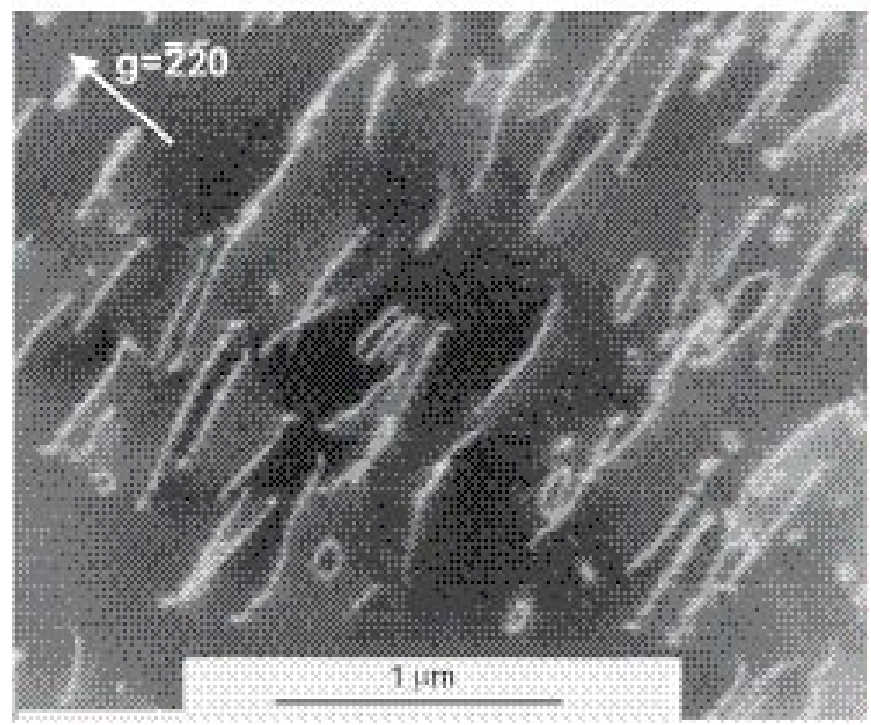

Fig. 3.- Micrografía de MET del plano primario (001) de una muestra con $24 \mathrm{~mol} \% \mathrm{Y}_{2} \mathrm{O}_{3}$ con un 3\% de deformación, mostrando subestructura de dislocaciones.

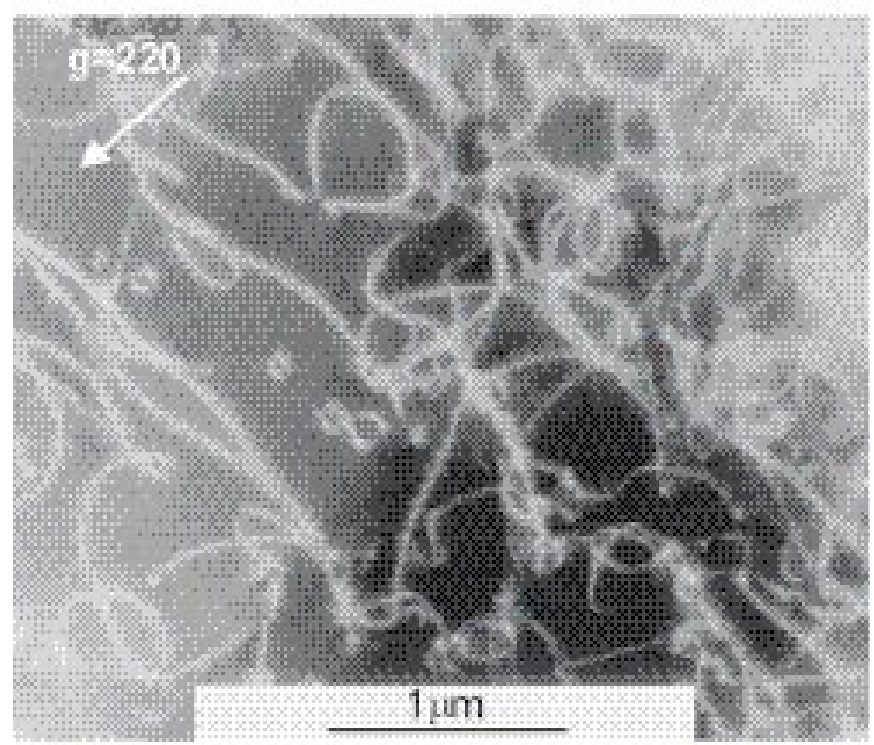

Fig. 4.- Micrografía de MET del plano primario de una muestra con $28 \mathrm{~mol} \% \mathrm{Y}_{2} \mathrm{O}_{3}$ deformada hasta un $8 \%$, que presentó inestabilidades plasticas, mostrando subestructura de dislocaciones. 


\section{DISCUSIÓN}

El modelo de ablandamiento térmico, desarrollado en (10) y propuesto por (9) para explicar las inestabilidades plásticas observadas se basa en un calentamiento adiabático local de la superficie del plano de deslizamiento. Este proceso implica la activación de una banda de deslizamiento cuya temperatura aumenta por la transformación en calor del trabajo desarrollado por las dislocaciones en su avance. Eventualmente, la banda queda inactiva debido al endurecimiento (work hardening), activándose otra en una región no deformada, y así sucesivamente hasta que la totalidad de la muestra haya experimentado deslizamiento primario. Es entonces cuando comenzaría el deslizamiento secundario (cross-slip). La ecuación que relaciona el trabajo necesario para la deformación durante una inestabilidad (W) con el incremento de temperatura en una banda $(\Delta \mathrm{T})$ es:

$$
\eta \mathrm{W}=\mathrm{C} \Delta \mathrm{T} \mathrm{V}_{\text {banda }}
$$

Donde $\eta \sim 0,9$ es la fracción de energía que se disipa como calor, $\mathrm{C}=3,5 \cdot 10^{-6} \mathrm{~J} \mathrm{~m}^{-3} \mathrm{~K}^{-1}$ es el calor específico de la circona y $\mathrm{V}_{\text {banda }}$ el volumen de la banda de deslizamiento activa, $\sim 20 \mu \mathrm{m}$. El trabajo que aparece en la ecuación anterior proviene de la deformación de la muestra, que puede escribirse como:

$$
\mathrm{W}=\sigma \mathrm{V} \Delta \mathcal{E}
$$

con $\sigma$ la tensión a la que ocurre la inestabilidad, $\mathrm{V}$ el volumen total de la muestra y $\Delta \varepsilon$ la deformación durante la inestabilidad. Hasta aquí el modelo de (9). Si consideramos sólo el término de deformación plástica, ya que la deformación elástica es despreciable durante una inestabilidad, tenemos que:

$$
\Delta \varepsilon=\dot{\varepsilon} \Delta t
$$

Suponiendo que el intervalo de tiempo considerado $\Delta \mathrm{t}$ es la duración típica de una inestabilidad (del orden de $40 \mathrm{~s}$ ), y sustituyendo los valores experimentales de nuestros ensayos mecánicos llegamos a que el incremento de temperatura en la banda $\Delta \mathrm{T} \sim 0,02 \mathrm{~K}$, lo cual es inconsistente con esta teoría, ya que es un valor demasiado pequeño que entra dentro del error experimental, y no da cuenta de las caídas de tensión observadas. Este valor contrasta con el obtenido por (11), $34 \mathrm{~K}$, que en sus cálculos no consideró la deformación plástica que ocurre durante una inestabilidad, sino sólo la elástica, y que asumió como tiempo típico de una inestabilidad $10^{-4} \mathrm{~s}$, valor tomado de la literatura para otro tipo de material, muy alejado de la realidad para la YCSZ.

La hipótesis de que se está produciendo un efecto Portevin-le-Chatelier (PLC) (12), sin embargo, se adecúa mucho más a los datos experimentales y es capaz de clarificar las características de las inestabilidades plásticas observadas. El efecto Portevin-le-Chatelier se origina cuando la velocidad de difusión de los defectos existentes en el material es del mismo orden que la de las dislocaciones al deslizar. En YCSZ, los defectos substitucionales de itrio, debido a su mayor radio iónico, son defectos puntuales que ejercen un importante efecto de talla durante la deformación plástica, lo cual ha sido puesto de manifiesto por algunos autores (13). Según el modelo de Cottrell $(12,14)$ una distribución inhomogénea de estos defectos alrededor de las dislocaciones, llamada atmósfera o nube de Cottrell, interacciona con ellas, frenando su movimiento. Sin embargo, para unas condiciones muy precisas de velocidad de deformación y temperatura, puede llegar a ocurrir que las dislocaciones alcancen una velocidad que les permita escapar momentáneamente de su nube de defectos, con lo que se produciría una avalancha de dislocaciones deslizando con gran rapidez, dando lugar a las caídas de tensión observadas experimentalmente. El efecto PLC se caracteriza por una insensibilidad de la tensión frente a cambios de velocidad de deformación y de temperatura, ya que es un fenómeno atérmico. Durante su desarrollo, pequeños cambios de velocidad de deformación o de temperatura (siempre que nos mantengamos en el rango adecuado para el fenómeno) no se traducen en aumentos o disminuciones de la tensión media, como cabría esperar, sino en cambios de frecuencia o amplitud de las oscilaciones. Al aumentar la velocidad impuesta a las dislocaciones, estas recorren un mayor trayecto al "escaparse", con lo cual aumenta la amplitud de las oscilaciones. Al aumentar la temperatura, los defectos difunden con mayor rapidez, su movilidad es más próxima a la de las dislocaciones, resultando más difícil el desbloqueo de éstas, con lo que disminuye la frecuencia de las oscilaciones. Experimentalmente, esto es lo que se produce en los ensayos de deformación, tal como puede verse en la figura 1. Aunque un análisis estadístico detallado de las oscilaciones, tal y como se hace en metales (15), aportaría información valiosa sobre el origen de las inestabilidades, un estudio de este tipo no ha sido posible debido a la baja frecuencia de las oscilaciones y a las limitaciones en cuanto a deformación tolerada por las cerámicas.

Otro hecho experimental que concuerda perfectamente con el fenómeno PLC son los incrementos de tensión, o "picos" que aparecen en las curvas tensión-deformación, como las de la figura 2 después de un recocido sin carga, una vez fuera del intervalo de condiciones PLC. Dicho incremento de tensión puede ser explicado porque durante el recocido, los defectos difunden hacia el corazón de las dislocaciones, aprisionándolas y produciendo un endurecimiento al comienzo del nuevo tramo de deformación plástica.

También podemos utilizar el modelo de Cottrell para estimar la velocidad que deben tener las dislocaciones para que se produzca el efecto PLC, y ver si coincide con la de nuestros ensayos de deformación. En el modelo de Cottrell aparecen tres regímenes en la deformación según la velocidad de las dislocaciones. Para velocidades muy lentas, la difusión de defectos controla la deformación. A partir de cierta velocidad de transición, $\mathrm{v}_{\mathrm{r}}$, se pasa a un régimen controlado por el deslizamiento de dislocaciones que interaccionan con los defectos. La interacción con los defectos es máxima para una velocidad crítica de las dislocaciones, $\mathrm{v}_{\text {c }}$. Cuando la velocidad de la dislocación es del mismo orden que la de difusión de los defectos, se produce el PLC. La velocidad de transición entre los dos regímenes mencionados, de difusión y de deslizamiento, fue calculada por (16), para la temperatura de $1400^{\circ}$. Según el modelo de Cottrell, la velocidad crítica de máxima interacción con las dislocaciones es aproximadamente un factor 10 mayor (12). Si aceptamos que, a grosso modo, el mismo factor separa las velocidades crítica y de PLC, nos encontramos con $\varepsilon \sim 4 \cdot 10^{-5} \mathrm{~s}^{-1}$, que coincide con las velocidades de deformación utilizadas en los ensayos mecánicos en que se han detectado inestabilidades plásticas. Por supuesto, las condiciones de velocidad de deformación y temperatura para la aparición de PLC constituyen una ventana muy precisa, y este modelo proporciona sólo una estimación grosera que, sin embargo, indica que el intervalo de velocidades de deformación en que se han encontrado inestabilidades es compatible con la velocidad de 
las dislocaciones durante el efecto PLC, apoyando nuestra hipótesis.

\section{CONCLUSIONES}

Se ha confirmado la existencia de inestabilidades plásticas en muestras de YCSZ con 21-24-28 mol\% de itria durante ensayos de deformación en compresión uniaxial para unas condiciones muy determinadas de velocidad de deformación ( $\dot{\varepsilon}$ entre $3-30 \cdot 10^{-4} \mathrm{~S}^{-1}$ ) y temperatura ( $\mathrm{T}$ entre $1310-1380^{\circ} \mathrm{C}$ ). El análisis, junto con una evaluación numérica del modelo de ablandamiento de bandas debido al calentamiento localizado, nos ha llevado a descartarlo por no ajustarse a los datos experimentales. Se ha puesto de manifiesto que los resultados de ensayos de deformación con cambios de velocidad de deformación, cambios de temperatura, recocidos sin carga, etc. son compatibles con un efecto PLC (Portevin-le-Chatelier), en el que las dislocaciones interaccionan con defectos puntuales de itrio. En particular, el hecho experimental de que cambios de temperatura y velocidad de deformación durante el ensayo no se traducen en cambios de la tensión media, y los efectos de endurecimiento tras recocidos sin carga fuera del régimen de inestabilidades, concuerdan perfectamente con una explicación de este tipo. Mediante el modelo de Cottrell se ha podido estimar (para la temperatura de los ensayos) la velocidad de deformación en la que se daría este fenómeno, siendo el resultado compatible con los datos experimentales.

\section{AGRADECIMIENTOS}

Este trabajo ha sido financiado por el proyecto de colaboración hispano-francesa HF2001-0125.

\section{BIBLIOGRAFÍA}

1. F. Wakai, S. Sakaguchi, and Y. Matsukno, "Superplasticity of yttria-stabilized tetragonal $\mathrm{ZrO}_{2}$ polycrystals", Adv. Ceram. Mater.,1, 259-63 (1986).

2. I.W. Chen and L.A. Xue, "Developement of superplastic structural ceramics", J. Amer. Ceram. Soc. 73, 2585 (1990)

3. A. Domínguez-Rodríguez, F. Guiberteau and M. Jiménez-Melendo; "Heterogeneous junction of yttria partially stabilized zirconia by superplastic flow", J. Mat. Res. 13, 1631 (1998).

4. D. Gómez-García , C. Lorenzo-Martín , A. Muñoz-Bernabé and A. Domínguez-Rodríguez, Model of high-temperature plastic deformation of nanocrystalline materials: Application to yttria tetragonal zirconia. Phys. Rev. B; 67, 144101 (2003).

5. A. H. Heuer, V. Lanteri and A. Domínguez-Rodríguez; "High temperature precipitation hardening of $\mathrm{Y}_{2} \mathrm{O}_{3}$ partially-stabilized zirconia single crystals", Acta Metall. 37, 559 (1989).

6. J. Martínez-Fernández, M. Jiménez-Melendo, A. Domínguez-Rodríguez, K.P.D. Lagerlöf and A. H. Heuer; "High Temperature precipitation hardening of $\mathrm{Y}_{2} \mathrm{O}_{3}$ partially-stabilized zirconia single crystals II. A quantitative model for the hardening", Acta Metall., 41, 3171 (1993).

7. D. Gómez-García, J. Martínez-Fernández, A.Domínguez-Rodríguez, P. Eveno, and J. Castaing, "Deformation Mechanisms for High-Temperature Creep of High Yttria Content Stabilized Zirconia Single Crystals", Acta Metall. Mater., 44, No. 3, 991-999 (1996).

8. A. Gallardo-López, J Martínez-Fernández, D. Gómez-García, A. DomínguezRodríguez, "High temperature plastic deformation of 24-32 mol\% yttria cubic stabilized zirconia (YCSZ) single crystals", J. Eur. Ceram. Soc., 23/13, 21832191 (2003).

9. K.J. McClellan, A.H. Heuer, L.P. Kubin, “Localized yielding during high temperature deformation of $\mathrm{Y}_{2} \mathrm{O}_{3}$-fully-stabilized cubic $\mathrm{ZrO}_{2}$ single crystals". Acta Metall. 44, [7], 2651-2662 (1996).

10. Estrin, Y. and Kubin, L.P., "Plastic instabilities: clasification and physical mechanisms", Res Mechanica, 23, 197-221 (1988).

11. McClellan, K. J., Structure/ Property Relations in $\mathrm{Y}_{2} \mathrm{O}_{3}$-Stabilized Cubic $\mathrm{ZrO}$ Single Crystals. PhD thesis, Case Western Reserve University, Cleveland, Ohio, U.S. (1994).

12. Friedel; Dislocations;. Pergamon Press, 368-84 (1964).

13. A. Domínguez-Rrodríguez, K.P.D. Lagerlöf and A.H. Heuer., "Plastic deformation and solid-solution hardening of $\mathrm{Y}_{2} \mathrm{O}_{3}$-stabilized $\mathrm{ZrO}_{2}$ ", J. Am. Ceram. Soc., 69, 281 (1986).

14. Cottrell, A.H., Dislocations and plastic flow in crystals. London. Oxford University Press (1953).

15. M.S. Bharathi, M. Lebyodkin, G. Ananthakrishna, C. Fressengeas, L.P. Kubin, "The hidden order behind jerky flow", Acta Mater., 50, 2813-2824 (2002).

16. A.Gallardo-López, D. Gómez-García, J. Martínez-Fernández, A. DomínguezRodríguez. "Influence of strain rate on the plastic deformation of high yttria content YCSZ single crystals at $1400-1500^{\circ} \mathrm{C}^{\prime \prime}$, Scripta Mater., 48, 1295 (2003). 


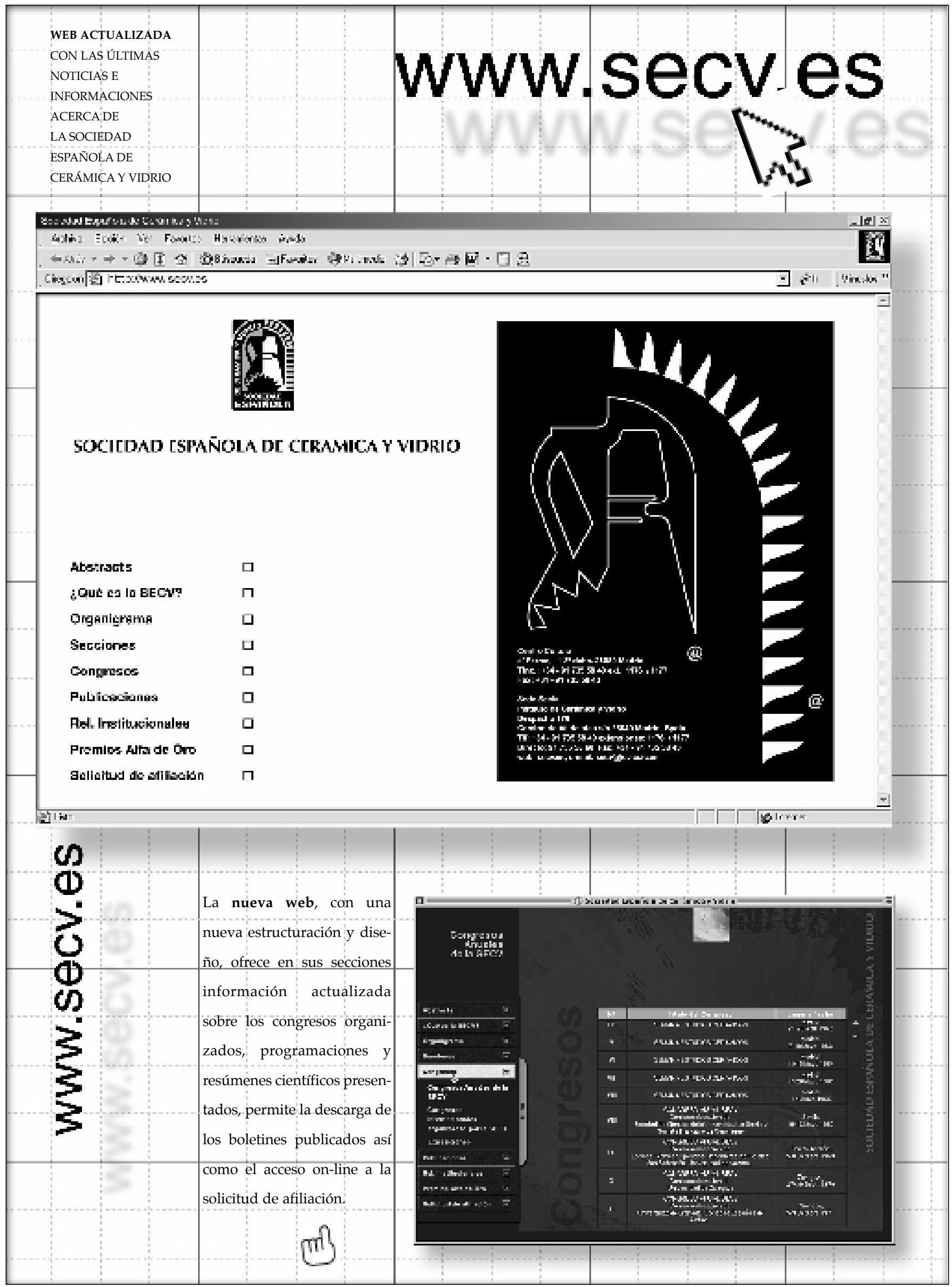

VISITE LA PÁGINA WEB DE LA SOCIEDAD ESPAÑOLA DE CERÁMICA Y VIDRIO EN HTTP://WWW.SECV.ES 